\title{
A Modified Naive Bayes to Online Training Assessment in Virtual Reality Simulators
}

\author{
Ronei Marcos de Moraes \\ Department of Statistics \\ Federal University of Paraíba, Brazil \\ ronei@de.ufpb.br
}

\author{
Liliane dos Santos Machado \\ Department of Informatics \\ Federal University of Paraíba, Brazil \\ liliane@di.ufpb.br
}

\begin{abstract}
Training systems based on virtual reality are used in several areas. In these systems the user is immersed into a virtual world to have realistic training through realistic interactions. In such training is important to know the quality of user's training. Several approaches to perform assessment in training simulators based on virtual reality have been proposed. However, they did not present a satisfactory solution for special cases as a bone marrow harvest in patients with bones deteriorated by disease. In this paper, we present an approach to online training assessment based on a Modified Naive Bayes, which can manipulate qualitative and quantitative variables simultaneously.
\end{abstract}

\section{Introduction}

Training systems based on virtual reality (VR) have been used in several areas [3]. The user is immersed into a virtual world to have realistic training and realistic interactions. However, it is important assess user's training to know the quality of his skills. Several kinds of training based on VR use to record the user actions in videotapes to post-analysis by experts [2]. In these cases, the user receives his assessment after some time. This is a problem because probably after some hours the user will not remember his exact actions what will make difficult the use of the assessment information to improve his performance. Besides of that, several kinds of training cannot be simply classified as bad or good due to its complexity. Then, the existence of an online assessment tool incorporated into a simulation system based on virtual reality is important to allow the learning improvement and users assessment [8]. As VR simulators are real-time systems, an assessment tool must continuously monitor all user interactions and compare his performance with pre-defined expert's classes of performance. By didactic reasons, the use of online assessment tools is more interesting once it can produce an assessment immediately after the training [15]. This kind of method allows the user to easily remember his mistakes and learn how to correct them.

In medicine, some models for online and offline assessment of training have been proposed $[5,6,7,8,10,11,12,14,15,16,17]$. The main problems related to online training assessment methodologies applied to VR systems are the computational complexity and the accuracy. An online assessment tool must have low complexity to do not compromise VR simulations performance, but it also must have high accuracy to do not compromise the user assessment. Another inconvenient about those methods is related to an unsatisfactory solution for specific cases as in some medical procedures, where there are quantitative and qualitative information available to perform an assessment. It was not found an assessment method compatible with these restrictions in the literature.

Some of the models previously mentioned are based on Naive Bayes (NB) or some modification of that $[17,19,20]$. NB can be adapted to analyze qualitative and quantitative information simultaneously and improve decision quality for special medical cases. In this paper, we propose a modified NB to lead with qualitative and quantitative variables simultaneously and can also assist the other requirements of an evaluation system for training based on VR.

\section{Virtual Reality and Simulated Training}

Virtual Reality refers to real-time systems modeled by computer graphics that allow user interaction and movements with three or more degrees of freedom $[3,24]$. More than a technology, VR became a new science that joins several fields as computers, robotics, graphics, engineering and cognition. VR worlds are 3D environments, created by computer graphics techniques, where one or more users are immersed totally or partially to interact with virtual elements. The 
realism of a virtual reality application is given by the graphics resolution and by the exploration of users senses. Mainly, special devices stimulate the sight, hearing and touch. As example, head-mounted displays (HMD) or even conventional monitors combined with special glasses can provide stereoscopic visualization, multiple sound sources positioned provides 3D sound, and touch can be simulated by the use of haptic devices [23].

Virtual reality systems for training can provide significant benefits over other methods of training, mainly in critical medical procedures. In some cases, those procedures are performed without any kind of visualization and the only information received is noticed by the touch sensations provided by a robotic device with force feedback. These devices can measure forces and torque applied during the user interaction [23] and these data can be used in an assessment [8,22]. A specific kind of haptic device, as the presented in Figure 1, is based on a robotic arm and provides force feedback and tactile sensations during user manipulation of objects in a three dimensional scene. This way, user can feel objects texture, density, elasticity and consistency. Since the objects have physical properties, a user can identify them in a $3 \mathrm{D}$ scene (without see them) by the use of this kind of device [23]. This is particularly interesting in medical applications to simulate proceedings in which visual information is not available. One of the main reasons for the use of such haptic devices in medical applications is their manipulation similarity when compared to real surgical tools.

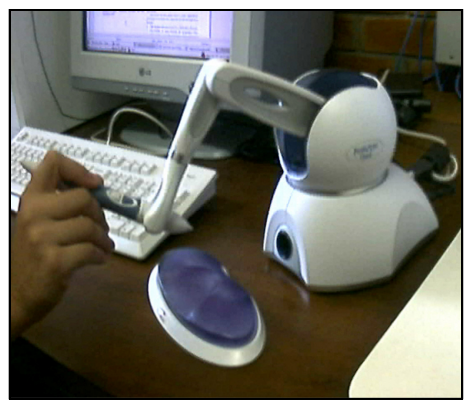

Figure 1. Haptic device used in VR systems.

\section{A Simulator for Bone Marrow Harvest Procedure}

This simulator has as goal to training new doctors to execute the bone marrow harvest, one of the stages of the bone marrow transplant. The procedure is done blindly, performed without any visual feedback, except the external view of the patient body. The physician needs to feel the skin and bone layers trespassed by the needle to find the bone marrow and then start the material aspiration (Figure 2). The simulator uses a robotic arm that operates with six degrees of freedom movements and provides force feedback to give to the user the tactile sensations felt during the penetration of the patient's body [9]. In the system, the robotic arm simulates the needle used in the real procedure, and the virtual body visually represented has the tactile properties of the real tissues.

In the bone marrow harvest, some special cases can occur and it can require specific skills of physician: in traditional transplant technique, multiple penetration are required (up to 20 per patient) in order to obtain enough bone marrow for that transplant. This procedure require specific applied force to trespass the bone and to reach the marrow, but to do not bypass it. For this reason, the repetitive procedures can cause loss of control or fatigue of physician. Another problem is the proximity between a first penetration and the next (generally about a centimeter), which require sufficient dexterity of the operator. In the pediatric oncology, it is easy to find children with soft bone structures or bones with deterioration caused by disease. In these cases, mistaken procedures can cause serious damages to the patient, as a fracture of the pelvic bone.

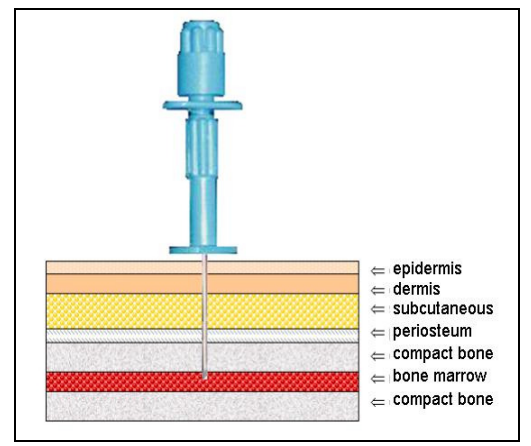

Figure 2. The tissue layers trespassed by needle in a bone marrow harvest.

The assessment tool proposed supervise the user movements during the puncture and evaluate the training according to $M$ possible classes of performance. The assessment of trainee is necessary to monitor the training quality and provide some feedback about the user performance. User movements, as spatial movements, can be collected from mouse, keyboard and any other tracking device. Applied forces, angles, position and torque can be collected from haptic devices [23]. Then, virtual reality systems can use one 
or more variables, as the mentioned above, to assess a simulation performed by user.

Specific assessment methods for bone marrow harvest procedure simulator have been proposed. Machado et al. [8] proposed the use of a fuzzy rulebased system. Moraes and Machado proposed several methods for online assessment $[10,15,16,17,18]$. After that, Morris et al. [21] suggested the use of statistical linear regression to evaluate user's progress in a bone surgery.

In this paper, we propose a new system to solve assessment problems in special cases of bone marrow harvest procedure, using a Modified Naive Bayes. This system is general and can perform an online training assessment in another kinds of virtual reality simulators too. The system uses a vector of information, with data collected from user interactions and positions with virtual reality simulator, and these data are compared by the assessment system with $M$ pre-defined classes of performance. To test the method proposed, we are using a bone marrow harvest simulator [9].

\section{Related Works}

This section presents a bibliographical review about methods for training assessment. For reader's better understanding, we first present a short review about the Naive Bayes method and in following, we present the Modified Naive Bayes.

\subsection{Naive Bayes Method}

The Naive Bayes (NB) method must be applied over discrete or multinomial variables $[1,17]$. Formally, let be the classes of performance in space of decision $\Omega=\{1, \ldots, M\}$ where $M$ is the total number of classes of performance. Let be $w_{i}, i \in \Omega$ the class of performance for an user. A Naive Bayes classifier computes conditional class probabilities and then predict the most probable class of a vector of training data $X$, according to sample data $D$, where $X$ is a vector with $n$ features obtained when a training is performed, i.e. $X=\left\{X_{1}, X_{2}\right.$, $\left.\ldots, X_{n}\right\}$. Using the Bayes Theorem:

$$
\begin{gathered}
P\left(w_{i} \mid X\right)=\left[P\left(X \mid w_{i}\right) P\left(w_{i}\right)\right] / P(X) \Leftrightarrow \\
\Leftrightarrow P\left(w_{i} \mid X_{1}, X_{2}, \ldots, X_{n}\right)= \\
=\left[P\left(X_{1}, X_{2}, \ldots, X_{n} \backslash w_{i}\right) P\left(w_{i}\right)\right] / P(X)
\end{gathered}
$$

However, as $P(X)$ is the same for all classes $w_{i}$, then it is not relevant for data classification and can be rewritten as:

$$
P\left(X \mid w_{i}\right) P\left(w_{i}\right)=P\left(X_{1}, X_{2}, \ldots, X_{n} \backslash w_{i}\right) P\left(w_{i}\right)
$$

The equation (2) is equivalent to the joint probability model:

$$
P\left(X_{1}, X_{2}, \ldots, X_{n} \backslash w_{i}\right) P\left(w_{i}\right)=P\left(X_{1}, X_{2}, \ldots, X_{n}, w_{i}\right)(3)
$$

Now, using successive applications of the conditional probability definition over equation (3), can be obtained:

$$
\begin{gathered}
P\left(X_{1}, X_{2}, \ldots, X_{n}, w_{i}\right)=P\left(w_{i}\right) P\left(X_{1}, X_{2}, \ldots, X_{n} \backslash w_{i}\right)= \\
=P\left(w_{i}\right) P\left(X_{1} \backslash w_{i}\right) P\left(X_{2}, \ldots, X_{n} \backslash w_{i}, X_{1}\right) \\
=P\left(w_{i}\right) P\left(X_{1} \backslash w_{i}\right) P\left(X_{2} \backslash w_{i}, X_{1}\right) P\left(X_{3}, \ldots, X_{n} \backslash w_{i}, X_{l},\right. \\
\left.X_{2}\right) \\
\ldots \\
=P\left(w_{i}\right) P\left(X_{1} \backslash w_{i}\right) P\left(X_{2} \backslash w_{i}, X_{1}\right) \ldots P\left(X_{n} \backslash w_{i}, X_{1},\right. \\
\left.X_{2}, \ldots, X_{n-1}\right)
\end{gathered}
$$

The Naive Bayes classifier receives this name because its naive assumption of each feature $X_{k}$ is conditionally independent of every other feature $X_{l}$, for all $\mathrm{k} \neq l \leq n$. It means that knowing the class is enough to determine the probability of a value $X_{k}$. This assumption simplifies the equation above, due to:

$$
P\left(X_{k} \backslash w_{i}, X_{l}\right)=P\left(X_{k} \backslash w_{i}\right)
$$

for each $X_{k}$ and the equation (3) can be rewritten as:

$$
\begin{gathered}
P\left(X_{1}, X_{2}, \ldots, X_{n}, w_{i}\right)= \\
=P\left(w_{i}\right) P\left(X_{1} \backslash w_{i}\right) P\left(X_{2} \backslash w_{i}\right) \ldots P\left(X_{n} \backslash w_{i}\right)
\end{gathered}
$$

unless a scale factor $\mathrm{S}$, which depends on $X_{1}, X_{2}, \ldots, X_{n}$. Finally, equation (1) can be expressed by:

$P\left(w_{i} \mid X_{1}, X_{2}, \ldots, X_{n}\right)=(1 / S) P\left(w_{i}\right) \Pi_{k=1}^{n} \quad P\left(X_{k} \backslash w_{i}\right)$

Then, the classification rule for Naive Bayes is done by:

$$
\begin{gathered}
X \in w_{i} \text { if } P\left(w_{i} \mid X_{1}, X_{2}, \ldots, X_{n}\right)>P\left(w_{j} \mid X_{1}, X_{2}, \ldots, X_{n}\right) \\
\quad \text { for all } i \neq j \text { and } i, j \in \Omega
\end{gathered}
$$

and $P\left(w_{*} \mid X_{1}, X_{2}, \ldots, X_{n}\right)$ with $*=\{i, j \mid i, j \in \Omega\}$, is done by (6).

To estimate parameters for $P\left(X_{k} \backslash w_{i}\right)$ for each class $i$, it was used a maximum likelihood estimator, named $P_{e}$ :

$$
P_{e}\left(X_{k} \backslash w_{i}\right)=\#\left(X_{k}, w_{i}\right) / \#\left(w_{i}\right)
$$


where $\#\left(X_{k}, w_{i}\right)$ is the number of sample cases belonging to class $w_{i}$ in all sample data $D$ and having the value $X_{k}, \#\left(w_{i}\right)$ is the number of sample cases that belong to the class $w_{i}$ in all sample data $D$.

\subsection{A Modified Naive Bayes}

As mentioned above, the NB method must be applied over discrete or multinomial variables. Some approaches were developed to use NB Method with continuous variables, as several discretization methods $[2,23]$ were used in the first stage to allow the use of the Naive Bayes method after. However, this approach can affect classification bias and variance of the NB method [25]. Other approach is use quantitative and qualitative variables simultaneously [4] and to compute its parameters from $D$. Formally, let be $X_{c a t}=\left\{X_{1}, X_{2}\right.$, $\left.\ldots, X_{c}\right\}, c(0 \leq c \leq n)$ categorical or discrete variables obtained from training data, as in classical Naive Bayes and $X_{c o n t}=\left\{X_{c+1}, X_{c+2}, \ldots, X_{n}\right\}, n-c$ continuous variables, obtained from training data too. Thus, $X$ is a vector with $n$ features, with $X=X_{\text {cat }} \cup X_{\text {cont }}$. Now, the equation (6) can be rewritten as [1]:

$$
\begin{gathered}
P\left(w_{i} \mid X_{1}, X_{2}, \ldots, X_{n}\right)= \\
=(1 / S) P\left(w_{i}\right) \prod_{k=1}^{c} \quad P\left(X_{k} \backslash w_{i}\right) \prod_{k=c+1}^{n} \quad P\left(X_{k} \backslash w_{i}\right)
\end{gathered}
$$

The equation (9) defines the Modified Naive Bayes (MNB) method. Categorical variables can be modeled by multinomial distributions. Discrete variables can be modeled by count of events in sample data $D$ or by a discrete statistical probability distributions. The continuous variables can be modeled by probability density functions. All these distributions models can be adjusted and verified by statistical tests over the data [13].

Based on the same space of decision with $M$ classes, a MNB method computes conditional class probabilities and then predicts the most probable class of a vector of training data $X$, according to sample data $D$. The parameters of MNB method are learning from data. The final decision about vector of training data $X$ is done by equation (7), where $P\left(w_{*} \mid X_{1}, X_{2}, \ldots, X_{n}\right)$ with $*=\{i, j \mid i, j \in \Omega\}$, is done by (9).

\section{Assessment Tool Assessment Based on Modified Naive Bayes}

The assessment tool proposed should supervise the user's movements and other parameters associated to them. The system must collect information about positions in the space, forces, torque, resistance, speeds, accelerations, temperatures, visualization position and/or visualization angle, sounds, smells and etc. The virtual reality simulator and the assessment tool are independent systems, however they act simultaneously. The user's interactions with the simulator are monitored and the information is sent to the assessment tool that analyzes the data and emits a report on the user's performance at the end of the training. Depending on the application, all those variables or some of them will be monitored (according to their relevance to the training).

The virtual reality system used for the tests is a bone marrow harvest simulator [9]. A special medical case was simulated: a child with soft bone structures. In a first movement on the real procedure, the trainee must feel the skin of the human pelvic area to find the best place to insert the needle used for the harvest. After, he must feel the tissue layers (epidermis, dermis, subcutaneous, periosteum and compact bone) trespassed by the needle and stop at the correct position to do the bone marrow extraction. As in this case the child has soft bone structures, the trainee must execute that step of procedure carefully to avoid damage to patient (a child, in this case). The simulator uses categorical variables as flags to identify the risk areas in the bone.

In VR simulator the trainee interacts with a robotic arm and his/her movements are monitored in the system by some variables [9]. For reasons of general performance of the VR simulator, were chosen to be monitored the following variables: spatial position, velocities, forces and time on each layer, as well as the risk areas in the bone. Previously, the system was calibrated by an expert, according $M$ classes of performance defined by him. The calibration process consists on execute several times the procedure and classify each one according to classes of performance. The number of classes of performance was defined as $M=3: 1)$ correct procedures, 2) acceptable procedures, 3) badly executed procedures. So, the classes of performance for a trainee could be: "you are well qualified", "you need some training yet", "you need more training".

The information of variability about these procedures was acquired using Modified Naive Bayes method. In this case, it was assumed that the font of information for $w_{i}$ classes is the vector of the sample data $D$. The trainee makes his/her training in virtual reality simulator and the Assessment Tool based on MNB collects the data from his/her manipulation. So, when a trainee uses the system, his/her performance is compared with each expert's class of performance and 
the Assessment Tool based on MNB assigns the better class, according to the trainee's performance. At the end of the training, the assessment system reports the classification to the trainee.

Table 1. Classification matrix for the Assessment Tool based on Modified Naive Bayes.

\begin{tabular}{|c|c|c|c|}
\hline \multirow{2}{*}{$\begin{array}{c}\text { Class of } \\
\text { performance } \\
\text { according to }\end{array}$} & \multicolumn{3}{|c|}{$\begin{array}{c}\text { Class of performance according to Assessment } \\
\text { Tool based on Modified Naive Bayes }\end{array}$} \\
\cline { 2 - 4 } & 1 & 2 & 3 \\
\hline 1 & 45 & 5 & 0 \\
\hline 2 & 8 & 36 & 6 \\
\hline 3 & 1 & 0 & 49 \\
\hline
\end{tabular}

The Kappa coefficient was $\mathrm{K}=80.0 \%$ with variance 0.001712 . The average of CPU time consumed for evaluation of training based on Modified Naive Bayes was lower than one millisecond of CPU using a Pentium Dual-Core PC compatible.

\section{Conclusions}

In this paper we presented a new approach for online training assessment in virtual reality simulators. This approach uses an Assessment Tool based on Modified Naive Bayes (MNB) and solves the main problems in assessment procedures: use of categorical (as risk areas in a soft bone), discrete and continuous variables simultaneously, low complexity and high accuracy.

A special medical case was simulated in bone marrow harvest simulator: a child with soft bone structures. The MNB was shown appropriate to evaluate that situation (according to Kappa coefficient), using categorical variables to monitor the risk areas in the bone, which can be fractured in case of mistaken procedure. Similar strategy can be used to monitor others special cases, as mentioned in this paper. Virtual reality based simulators, designed for other areas also can use this approach to assess specific skills of users.

\section{Acknowledgment}

This work is partially supported by Brazilian Council for Scientific and Technological Development, CNPq (Process 303444/2006-1) and Brazilian Research and Projects Financing, FINEP (Grant 01-041054-000).

\section{References}

[1] Borgelt, C. and Kruse, R., Graphical Models: Methods for Data Analysis and Mining. Wiley, 2002.

[2] G. Burdea, G. Patounakis, V. Popescu, and R.E. Weiss. "Virtual Reality Training for the Diagnosis of Prostate Cancer". Proc. IEEE Virtual Reality Annual Int. Symposium, pp. 190-197, 1998.

[3] Burdea, G. and Coiffet, P., Virtual Reality Technology. 2nd ed., Wiley Interscience, 2003.

[4] C. Doring, C. Borgelt, and R. Kruse. "Fuzzy clustering of quantitative and qualitative data", Proc. of the 2004 NAFIPS, pp. 84-89, 2004.

[5] M. Färber et al. "Heinz Handels Training and evaluation of lumbar punctures in a VR-environment using a 6DOF haptic device", Studies in Health Tech. and Informatics, v. 132, pp. 112-114, 2008.

[6] J. Huang, S. Payandeh, P. Doris, and I. Hajshirmohammadi, "Fuzzy Classification: Towards Evaluating Performance on a Surgical Simulator". Studies in Health Technology. and Informatics, v. 111, pp. 194-200, 2005.

[7] K. Kumagai, J. Yamashita, O. Morikawa, and K. Yokoyama, "A New Force-Based Objective Assessment of Technical Skills in Endoscopic Sinus Surgery". Studies in Health Technology and Informatics, v.125, pp. 235-237, 2007.

[8] L.S. Machado, R.M. Moraes, and M.K. Zuffo, "Fuzzy Rule-Based Evaluation for a Haptic and Stereo Simulator for Bone Marrow Harvest for Transplant", Proc. 5th Phantom Users Group Workshop, 2000.

[9] L.S. Machado et al., "A Virtual Reality Simulator for Bone Marrow Harvest for Pediatric Transplant”. Studies in Health Technology and Informatics, v. 81, pp .293-297, 2001.

[10] L.S. Machado and R.M. Moraes, "Online Training Evaluation in Virtual Reality Simulators Using Evolving Fuzzy Neural Networks", Proceedings of the 6th Int. FLINS Conf., Belgium., pp. 314-317, 2004.

[11] T. Mackel, J. Rosen, and C. Pugh, "Data Mining of the E-Pelvis Simulator Database: A Quest for a Generalized Algorithm for Objectively Assessing Medical Skill", Studies in Health Technology and Informatics, v. 119, pp. 355-360, 2006.

[12] T. Mackel, J. Rosen, and C. Pugh, "Application of Hidden Markov Modeling to Objective Medical Skill Evaluation". Studies in Health Technology and Informatics, v. 125, pp. 316-318, 2007. 
[13] Mood, A. M., Graybill, F., and Boes, D. C.; Introduction to the Theory of Statistics. McGraw-Hill, 3rd ed., 1974.

[14] R.M. Moraes and L.S. Machado, “ Fuzzy Gaussian Mixture Models for On-line Training Evaluation in Virtual Reality Simulators". Annals of the Int. Conference on Fuzzy Information Processing (FIP'2003), March, China, v.2, pp. 733-740, 2003.

[15] R.M. Moraes and L.S. Machado, "Using Fuzzy Hidden Markov Models for Online Training Evaluation and Classification in Virtual Reality Simulators", Int. Journal of General Systems, v.33 n.2-3, pp. 281-288, 2004.

[16] R.M. Moraes and L.S. Machado, "Maximum Likelihood for On-line Evaluation of Training Based on Virtual Reality", Proc. Global Congress on Engineering and Tech. Education (GCETE,2005), March, Brazil, pp. 299-302, 2005.

[17] R.M. Moraes and L.S. Machado, "Assessment Based on Naive Bayes for Training Based on Virtual Reality", Proc. Int. Conf. on Engineering and Computer Education (ICECE'2007), March, Brazil, pp. 269-273, 2007.

[18] R.M. Moraes and L.S. Machado, "Fuzzy Bayes Rule for On-Line Training Assessment in Virtual Reality Simulators", Journal of Multiple-Valued Logic and Soft Computing, v.14, n.3-5, pp.325-338, 2008.
[19] R.M. Moraes and L.S. Machado, "Online Training Assessment in Virtual Reality Simulators Based on Fuzzy Naive Bayes", Proc. of International Conference on Engineering and Technology Education 2008 (Intertech'2008). March, Brazil, pp. 145-149, 2008.

[20] R.M. Moraes and L.S. Machado, "Online Training Assessment in Virtual Reality Simulators Based on Gaussian Naive Bayes". 8th International FLINS Conf., September, Spain. 2008. (to appear).

[21] D. Morris et al., "Visuohaptic simulation of bone surgery for training and evaluation". IEEE Computer Graphics and Applications, v.26, n.6, 2006, pp. 48-57.

[22] J. Rosen, C. Richards, B. Hannaford, and M. Sinanan, "Hidden Markov Models of Minimally Invasive Surgery", Studies in Health Tech. and Informatics, v. 70, pp. 279-285, 2000.

[23] K. Salisbury, "Haptics: The Technology of Touch", HPCWire Special. n. 10. Nov. 1995.

[24] Vince, J. Virtual Reality Systems. Addison-Wesley, 1995.

[25] Y. Yang and F.I. Webb, "On Why Discretization Works for Naive-Bayes Classifiers", Lecture Notes on Artificial Intelligence, v. 2903, pp. 440-452, 2003. 